\title{
LAS AMENAZAS Y LA SIMPATÍA HACIA CANDIDATOS AUTORITARIOS: EVIDENCIA DE PERÚ
}

\author{
Threats and Sympathy for Authoritarian Candidates: \\ Evidence from Peru
}

\author{
As ameaças e a simpatia pelos candidatos autoritários: \\ evidência do Peru
}

\section{Arturo MALDONADO}

Pontificia Universidad Católica del Perú. Lima, Perú. Email: arturo.maldonado@pucp.pe (D)

Envío: 2020-03-19

Aceptado: 2020-08-16

First View: 2020-10-13

Publicado: 2020-11-30

\begin{abstract}
Palabras clave:
Resumen autoritarismo; amenaza

Este artículo evalúa si la relación entre las predisposiciones autoritarias y la simpatía por candidatos autoritarios se amplifica en presencia de amenazas econóeconómica; amenaza por la delincuencia; elecciones; Perú micas o por la delincuencia y el crimen. El análisis se realiza sobre datos de una encuesta aplicada después de la primera vuelta de la última elección presidencial de 2016 en Perú que incluye la simpatía hacia tres candidatos: Keiko Fujimori, percibida como autoritaria, y Pedro Pablo Kuczynski y Verónika Mendoza, considerados como no autoritarios. El instrumento incluyó un experimento de viñetas con noticias sobre economía y delincuencia, fraseadas de tal manera que enfatizaran las amenazas sobre estos temas. En esta encuesta también se midió el autoritarismo individual a través de las actitudes hacia la crianza de los niños. Los resultados indican que los ciudadanos más autoritarios, en situaciones de amenaza, sobre todo económica, manifiestan una mejor valoración de una candidata autoritaria como Keiko Fujimori. Este efecto no se detecta para los candidatos no autoritarios que competían en estas mismas elecciones.
\end{abstract}




\section{Keywords:} authoritarianism; economic threat; crime threat; elections; Peru

\begin{abstract}
This article assesses whether the relationship between authoritarian predispositions and sympathy for authoritarian candidates is amplified in the presence of economic or security threats. The analysis is carried out on data from a national survey applied after the first round of the last presidential election of 2016 in Peru that included measures of sympathy for three candidates: Keiko Fujimori, perceived as authoritarian, and Pedro Pablo Kuczynski and Verónika Mendoza, considered non-authoritarian candidates. The instrument included a vignette experiment with news about the economy and crime, worded in such a way as to emphasize the threats about these issues. This survey also measured individuals' authoritarianism through attitudes towards child rearing. Results indicate that in threatening situations, especially economic ones, the most authoritarian citizens more greatly value an authoritarian candidate like Keiko Fujimori. This effect is not detected for nonauthoritarian candidates competing in this election.
\end{abstract}

Palavras-chave: autoritarismo; ameaça econômica; ameaça de crime; eleições; Peru

\section{Resumo}

Este artigo avalia se a relação entre predisposições autoritárias e simpatia por candidatos autoritários é ampliada na presença de ameaças econômicas ou pela delinquência e crime. A análise é feita com base em dados de uma pesquisa aplicada após o primeiro turno da última eleição presidencial de 2016 no Peru, que inclui simpatia por três candidatos: Keiko Fujimori, considerada autoritária, e Pedro Pablo Kuczynski e Verónika Mendoza, considerados não autoritários. $\bigcirc$ instrumento incluía uma vinheta de experimento com notícias sobre economia e criminalidade, formulada de uma maneira que enfatizava as ameaças sobre essas questões. Essa pesquisa também mediu o autoritarismo individual por meio de atitudes em relação à educação dos filhos. Os resultados indicam que os cidadãos mais autoritários, em situações ameaçadoras, principalmente econômicas, mostram uma melhor avaliação de um candidato autoritário como Keiko Fujimori. Este efeito não é detectado para candidatos não autoritários que competem nessas mesmas eleições.

\section{INTRODUCCIÓN}

La relación entre autoritarismo y elecciones ha cobrado actualidad debido a los resultados sorpresivos de algunos procesos internacionales. Entre estos destaca la victoria presidencial de Donald Trump en los Estados Unidos en 2016, que es considerado como un líder autoritario (Nordland, 2017). Otros ejemplos van desde la victoria de Viktor Orbán en Hungría (Buzogány, 2017) a la de Recep Erdogan en Turquía (Benhabib, 2013). En América Latina, por el contrario, las elecciones han dado como resultado el surgimiento de caudillos y de personajes autoritarios con mayor frecuencia a lo largo de la historia. La literatura presenta ejemplos del resurgimiento de regímenes autoritarios, dando lugar a una ola reversa de democratización (Haynes, 2001), lo que ha dado lugar a la aparición de estudios en donde se evalúa la elección de líderes autoritarios a través de mecanismos de la democracia representativa (Levitsky y Way, 2010). 
¿Qué lleva a los ciudadanos de un país a apoyar a candidatos con propuestas autoritarias?

La literatura ha asociado las actitudes autoritarias con el apoyo a medidas antidemocráticas y de mano dura, principalmente en los países industrializados (Hetherinton y Suhay, 2011; Roccato, Vieno y Russo, 2014) y, más recientemente, en América Latina (Azpuru y Malone, 2018). Un trabajo más cercano a esta propuesta asocia el autoritarismo con el voto hacia líderes con tendencias iliberales en América Latina (Cohen y Smith, 2016). Todos ellos asumen que el autoritarismo en los individuos es un rasgo de la personalidad, casi constante en el tiempo, con un efecto permanente en otras actitudes y comportamientos. Sin embargo, otra rama de la literatura propone que el autoritarismo individual es una predisposición latente que puede ser activada bajo la presencia de amenazas (Feldman y Stenner, 1997). Es decir, según esta perspectiva, los ciudadanos expresan actitudes y comportamientos más punitivos y etnocéntricos, menos tolerantes y más propensos a opiniones autoritarias cuando perciben graves amenazas, como crisis económicas o atentados terroristas. Por el contrario, en situaciones en donde la percepción de peligros no está presente pueden no expresar estas actitudes y opiniones, e incluso no tener consecuencias visibles en el comportamiento. Se puede inferir, entonces, que los ciudadanos que perciben amenazas severas en su entorno en periodos electorales podrían canalizar estas actitudes y opiniones mediante sus simpatías electorales o apoyo a sus líderes.

En este trabajo partimos de la literatura que indica que las predisposiciones autoritarias juegan un papel importante en las simpatías hacia este tipo de candidatos. Pero, yendo un paso más allá, se propone que las amenazas activan el efecto de estas predisposiciones hacia líderes postulantes que desafían los mecanismos de la democracia liberal. Con este fin se replica una medición del autoritarismo por medio de indicadores de los valores en la crianza de los niños, y se propone un diseño experimental, donde se utilizan amenazas económicas y a la seguridad ciudadana para activar la relación entre las predisposiciones autoritarias y la simpatía hacia los distintos candidatos.

Las últimas elecciones presidenciales de 2016 en Perú ofrecen una oportunidad para evaluar las simpatías hacia Keiko Fujimori, una candidata percibida como autoritaria, frente a otros candidatos que no presentan este perfil, como Kuczynski y Mendoza. El contexto peruano, además, es propicio para trabajar con amenazas económicas y a la seguridad, dada la historia de colapso económico y aumento de la criminalidad en el país. Estos temas aparecen frecuentemente en el grupo de principales problemas del país (Carrión et al., 2018). 


\section{AUTORITARISMO Y ELECCIONES}

El concepto de autoritarismo ha tenido un largo viaje que se inicia en la década del 50 del siglo pasado con los estudios pioneros de Fromm (1941) sobre la emergencia del fascismo o los de Adorno (1950) sobre la personalidad autoritaria. Janowitz y Marvick (1953) definen a una persona autoritaria como «el individuo que se preocupa por el poder y la fortaleza, y que es propenso a resolver los conflictos de manera arbitraria» (p. 185).

Más recientemente, Altemeyer (1996) diferencia 3 dimensiones de este concepto a nivel de los individuos: la sumisión a la autoridad, la agresión autoritaria y el convencionalismo. Se entiende por sumisión autoritaria el respeto y la obediencia necesarios para respaldar el orden social establecido. La agresión, según Feldman, es «la hostilidad dirigida hacia quienes son vistos como una amenaza al orden social» (2003, p. 67). Finalmente, el convencionalismo alude a la preferencia por las propias costumbres y tradiciones y por una desvaloración de otras convenciones sociales.

Los ciudadanos autoritarios podrían expresar sus opciones agresivas mediante las preferencias por políticas de mano dura y buscarían líderes fuertes que encarnen esta necesidad de orden y autoridad. Esta línea de investigación propone una conexión directa entre las predisposiciones autoritarias y la preferencia por un tipo de liderazgo en particular, como el caso de Trump en Estados Unidos (Federico, Johnson y Lavine, 2016; Lakoff, 2016; Taub, 2016) o el apoyo a gobiernos o líderes autoritarios en América Latina (Stevens, Bishin y Barr, 2006; Cohen y Smith, 2016). En el caso concreto del Perú existen trabajos que relacionan este concepto con el apoyo a ciertos políticos. Se encuentra que individuos que exhiben estos rasgos suelen votar más a favor de Keiko Fujimori, una candidata considerada a la derecha en el espectro ideológico, y menos a favor de Ollanta Humala, candidato ubicado a la izquierda (Rottenbacher, 2012; Christiansen, 2016). En general, estos trabajos evalúan la relación directa entre estas actitudes y el apoyo electoral a líderes con tendencias iliberales. Desde esta perspectiva, el autoritarismo es visto como un rasgo de la personalidad de los individuos, en línea con la propuesta teórica de Altemeyer (1996).

Sin embargo, desarrollos teóricos alternativos entienden este concepto como una predisposición latente, cuyas manifestaciones son activadas por contextos de amenazas (Feldman, 2003; Feldman y Stenner, 1997). Estas manifestaciones pueden ser afectivas, cognitivas o de comportamiento, observables mediante opiniones agresivas o punitivas, intolerancia hacia minorías o preferencias de mano dura (Stenner, 2005). En ausencia de amenazas, no se observarían estas actitudes y comportamientos o se reportarían con una menor intensidad. Es decir, este modelo propone que las amenazas moderan la relación entre el autoritarismo y las preferencias políticas y sociales (Lavine, Lodge y Freitas, 2005). 
En muchos casos el mecanismo causal que vincula las amenazas con la activación de las manifestaciones autoritarias es uno emocional. Feldman y Stenner (1997) mencionan que la ansiedad provocada por las amenazas es el motor del autoritarismo. La ansiedad que generan la economía o el crimen, por ejemplo, lleva a los ciudadanos de América Latina a actitudes punitivas (Singer et al., 2019). Este mecanismo emocional puede generar la búsqueda de líderes que prometan restaurar el orden establecido y, por lo tanto, eliminar la incertidumbre y la ansiedad.

Los tipos de amenazas que se han evaluado en la literatura son diversos, pero aluden a elementos desestabilizadores del orden social o de la propia estabilidad, como, por ejemplo, el desempleo, el incremento de la criminalidad, los desórdenes civiles, los atentados terroristas o las guerras (Feldman y Stenner, 1997).

\section{DELINCUENCIA Y ECONOMÍA COMO AMENAZAS EN PERÚ}

La delincuencia y la economía son amenazas notables que colocan a una amplia franja de ciudadanos peruanos en una situación de vulnerabilidad. En ambos casos, la realidad presenta indicadores desalentadores: delincuencia al alza y economía frágil.

La delincuencia ha desplazado a la economía como el principal problema reportado por los ciudadanos peruanos. Perú se encuentra entre los países con más alto nivel de victimización por delincuencia -33\%-y en percepción de inseguridad ciudadana en las Américas -56.7 puntos en una escala del 0 al 100- (Carrión et al., 2018), con un incremento sostenido de las denuncias policiales y una escasa confianza en las instituciones de la seguridad y la justicia (Costa y Romero, 2014).

A pesar de la estabilidad económica de las últimas décadas en Perú, la economía todavía es un tema que genera ansiedad entre los ciudadanos peruanos. De hecho, todavía una proporción importante de personas lo considera el problema más importante del país -21.4 \%- (Carrión et al., 2018). Esta situación se agrava considerando que el crecimiento macroeconómico peruano (y de América Latina en general) ha generado un aumento de la clase media, que incluye una clase media vulnerable, propensa a volver a una situación de pobreza ante shocks como crisis económicas o enfermedades (Stampini et al., 2015; Jaramillo y Zambrano, 2013).

Las amenazas sobre la economía y el crimen podrían tener efectos diferenciados. Se podría esperar que la delincuencia tenga un efecto mayor debido a la preocupación ciudadana por el tema. También se podría argumentar que la economía tendría un efecto más importante debido a las crisis económicas históricas vividas por los peruanos. Sin embargo, en la literatura, las amenazas económicas y a la seguridad aparecen interrelacionadas. Dammert y Malone (2003) encuentran que el amplio miedo al crimen en Chile sería una manifestación de otras inseguridades, 
como las económicas, relacionadas con el desempleo, la inflación y la pérdida de ingresos.

En este estudio, se evalúa el efecto condicionante de ambos issues con experimentos separados, asumiendo que pueden tener efectos diferenciados, pero reconociendo que ambas amenazas están interrelacionadas.

\section{CONSECUENCIAS ELECTORALES DEL AUTORITARISMO ACTIVADO}

En este trabajo se parte del autoritarismo individual, concepto ligado con la sumisión a la autoridad y a las normas del grupo, y se lo relaciona con el «autoritarismo institucional». Este último concepto, como lo definen Cohen y Smith (2016), está relacionado con gobernantes que, pudiendo haber sido elegidos en elecciones libres y justas, constantemente socavan los derechos y las libertades ciudadanas, remueven los controles y amenazan el balance de poderes.

Así como se puede clasificar a los gobernantes según el tipo de políticas que implementan, también se puede clasificar a los candidatos a presidente según el tipo de ofrecimientos que hacen en campaña. En este trabajo usaremos los ofrecimientos de los candidatos a la presidencia en Perú en el 2016 en lo que respecta a seguridad ciudadana como una forma de clasificarlos como autoritarios o no, según ofrezcan políticas de mano dura (por ejemplo, militarización de la seguridad ciudadana o pena de muerte para delitos graves) u otro tipo de ofertas no punitivas.

Cohen y Smith (2016) encuentran que los ciudadanos autoritarios (los que son sumisos a la autoridad y que tienen conformidad a las normas del grupo social) tienden a votar por candidatos autoritarios (que desafían a las instituciones liberales y proponen medidas de mano dura) de derecha. Este trabajo no solo evalúa el efecto directo de estas predisposiciones en el apoyo a candidatos, más cercano a la idea teórica del autoritarismo como rasgo de la personalidad, sino su efecto moderado por las amenazas en la simpatía hacia candidatos autoritarios, más cercano a la propuesta teórica del autoritarismo como predisposición latente.

De acuerdo a la literatura presentada, este estudio plantea la siguiente relación entre variables, tal y como se muestra en la Figura 1. La hipótesis que se evaluará en este artículo es:

H1: El tratamiento de amenaza económica y a la seguridad amplificará el efecto de las predisposiciones autoritarias en la simpatía hacia candidatos con propuestas de mano dura.

En ausencia de amenazas, podrá existir un efecto directo del autoritarismo en la simpatía hacia candidatos de mano dura, pero sería menor o no significativo. 
Figura 1. Relación entre variables

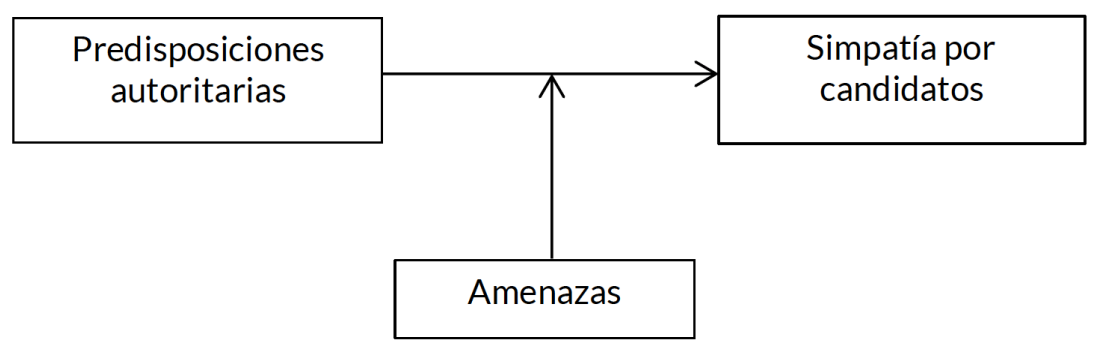

Fuente: elaboración propia.

\section{POSICIONAMIENTOS DE LOS CANDIDATOS EN EL PROCESO ELECTORAL DE 2016}

En las últimas elecciones presidenciales de 2016 en Perú, algunos candidatos ofrecieron medidas de mano dura para lidiar con la delincuencia. Estas propuestas son características de candidatos considerados autoritarios, es decir, de aquellos que plantean medidas que están cercanas al uso discrecional o abusivo del poder. Un ejemplo de ello fueron las propuestas de Keiko Fujimori, candidata de Fuerza Popular, partido considerado de derecha conservadora, quien se manifestó a favor de la construcción de penales factoría para menores de edad y de cárceles arriba de los cuatro mil metros sobre el nivel del mar para encerrar a los delincuentes de alta peligrosidad (Gestión, 2016).

Pedro Pablo Kuczynski, candidato por Peruanos por el Kambio, organización de derecha tecnocrática, y electo presidente, manifestó durante la campaña que él estaba en contra de sacar a los militares a la calle como medida de protección frente a la delincuencia. Ante esta posición, Fujimori manifestó durante la campaña: «Él no tiene las cosas claras. No tiene la mano firme para luchar contra este flagelo. Yo me ratifico. A mí no me temblará la mano, tengo los pantalones bien puestos» (Lolas, 2016). Es decir, Kuczynski y Fujimori se ubicaban en una posición de derecha, pero diferían en sus posiciones respecto a temas de seguridad ciudadana, Fujimori más afín a medidas punitivas y Kuczynski a medidas preventivas.

Verónika Mendoza era la candidata del Frente Amplio, coalición de organizaciones de izquierda, que proponía en temas de seguridad ciudadana el reforzamiento de las labores de inteligencia, el fortalecimiento operativo de la policía y el énfasis en las oportunidades para los infractores de la ley. La candidata estaba claramente en contra de propuestas de mano dura para combatir la delincuencia y el crimen organizado, como encargar a los militares la lucha contra la delincuencia o como la pena de muerte (La República, 2016). Es decir, Mendoza compartía con Kuczynski una posición opuesta a la mano dura encarnada por Fujimori. Sin 
embargo, Mendoza se ubicaba en una posición de izquierda, contraria a Kuczynski y Fujimori, ambos a la derecha.

En el aspecto económico, Kuczynski fue claramente el candidato que más se identificaba con el modelo económico por sus posiciones programáticas y por su carrera como exbanquero de inversión y ministro de Economía durante la gestión presidencial de Alejandro Toledo. Sus ofertas de campaña giraban en torno a disminuir la informalidad, brindando beneficios a las empresas (sobre todo las pequeñas) para que contraten trabajadores formalmente y en el fomento de las inversiones privadas (Gestión, 2015). En general, se trataba de propuestas para afianzar el modelo económico, no para cuestionarlo.

Keiko Fujimori en el plano económico era percibida de una manera muy similar que Kuczynski, aunque algunos expertos la ubicaban en una posición más centrista, entre los extremos que representaban Kuczynski y Mendoza (Paredes, 2016). Siendo la hija del gobernante que inauguró el modelo económico en Perú a comienzos de los noventa, Keiko Fujimori también representaba la continuidad del modelo, con modificaciones que no pusieran en duda su vigencia.

Mendoza sí representaba una posición crítica al modelo económico y ofrecía un cambio a las políticas económicas neoliberales. Algunos periodistas opinaban que la oferta económica de Mendoza era peligrosa, debido a que implicaba un mayor gasto estatal financiado con impuestos, la desdolarización del sistema bancario, el control de precios de alimentos mediante subsidios, la reforma del sistema de pensiones, el aumento de la remuneración mínima vital y la renegociación de contratos con empresas extractivas y de los tratados de libre comercio (Palacios, 2016).

Estas posiciones, tanto en lo económico como en lo relativo a la seguridad, eran reconocidas por los electores. De hecho, no se observaban diferencias en la ubicación en la escala izquierda-derecha o en el continuo mercado-Estado entre Kuczynski y Fujimori, entre los peruanos. Donde sí se apreciaban contrastes entre estos candidatos era en un continuo ley-discrecionalidad, donde Kuczynski era visto como el candidato más respetuoso de la ley. En estos continuos, Mendoza era considerada una candidata de izquierda, que privilegia el Estado sobre el mercado y con una tendencia a la discrecionalidad (Sulmont, 2018). Es más, según una encuesta realizada en mayo de 2016, en la cual se les pidió a los entrevistados que señalaran cuál es el candidato que tendría un estilo de gobierno de más mano dura, el 56 \% creía que sería Fujimori mientras que el 37 \% creía que Kuczynski gobernaría con ese estilo. En otra medición de abril de 2016, los electores ubicaban a Kuczynski en el cuadrante democrático ${ }^{1}$ de derecha ${ }^{2}$ y a Keiko Fujimori en el cuadrante autoritario de derecha (GFK, 2016).

1. Usando una escala del 1 al 7 , donde 1 es autoritario y 7 es democrático y marcando el punto de corte en 4.

2. Usando una escala del 1 al 7 donde 1 es izquierda y 7 es derecha y marcando el punto de corte en 4. 
Vemos, entonces, que la elección presidencial en Perú en 2016 presentaba la oportunidad de evaluar la activación del efecto del autoritarismo mediante amenazas en las simpatías hacia una candidata autoritaria como Keiko Fujimori y hacia candidatos no autoritarios como Kuczynski y Mendoza, aunque estos últimos en posiciones ideológicas opuestas.

\section{DATOS, METODOLOGÍA Y MEDICIÓN DE AUTORITARISMO}

Los datos con los que se trabaja en este estudio provienen de una encuesta electoral aplicada a hombres y mujeres de 18 años o más, electores hábiles de 19 regiones del Perú, realizada en mayo de 2016, luego de la primera vuelta, pero antes del ballotage. La selección de observaciones fue probabilística, polietápica y estratificada según región y ámbito urbano y rural. Las zonas donde se realizaron las entrevistas concentran aproximadamente el $65 \%$ de la población nacional mayor de edad. Esta base de datos cuenta con 1572 observaciones.

En esta encuesta se introdujo la medición de las actitudes autoritarias en la crianza de los niños, que es una medida de autoritarismo usada en la literatura. Por ejemplo, la American National Election Study y la World Values Survey incluyen esta medición en sus cuestionarios (Norris e Inglehart, 2017). Esta aproximación al autoritarismo ha sido utilizada también por Cohen y Smith (2016) y por Azpuru y Malone (2018) en América Latina ${ }^{3}$.

Esta medición del autoritarismo en la crianza de los niños implica que los entrevistados respondan acerca de sus preferencias por valores que preferían para inculcar a los niños. A cada entrevistado se le pedía que reportara sus preferencias en los siguientes pares de opciones: (1) un(a) niño(a) debe obedecer a sus padres o un(a) niño(a) debe ser responsable por sus propias acciones; (2) un(a) niño(a) debe tener respeto por sus mayores o un(a) niño(a) debe pensar por sí mismo; y (3) un(a) niño(a) debe tener buenos modales o un(a) niño(a) debe tener sentido común y buen juicio.

Con estas preguntas se creó un índice de actitudes autoritarias ${ }^{4}$. A las respuestas de "obedecer», «respeto por sus mayores» $y$ «buenos modales» se les asignó un

3. El autoritarismo de ala derecha (RWA) es otra medición que se usa frecuentemente en la literatura. Sin embargo, esta opción mide las actitudes autoritarias y sus expresiones conductuales, pero no se aproxima a las predisposiciones individuales al autoritarismo. En ese sentido, es posible que el RWA no identifique a los individuos autoritarios que no han sido activados por las amenazas. La medición de autoritarismo a través de los valores de crianza en los niños permite distinguir las predisposiciones autoritarias de los productos autoritarios (MacWilliams, 2016).

4. El respectivo Alpha de Cronbach para estos tres ítems es 0,517. Cohen y Smith (2016) reportan los respectivos coeficientes de Alpha de Cronbach para esta batería en 18 países de América Latina, donde el coeficiente de la región es 0,46 y el de Perú es 0,39. 
valor de 1, de tal manera que el índice varía entre 0 (aquellos que no seleccionaron ninguna de estas opciones) hasta 3 (aquellos que seleccionaron estas tres opciones). La Figura 2 presenta el porcentaje y el número de entrevistados por cada uno de los valores del índice.

Figura 2. Distribución del índice de actitudes autoritarias

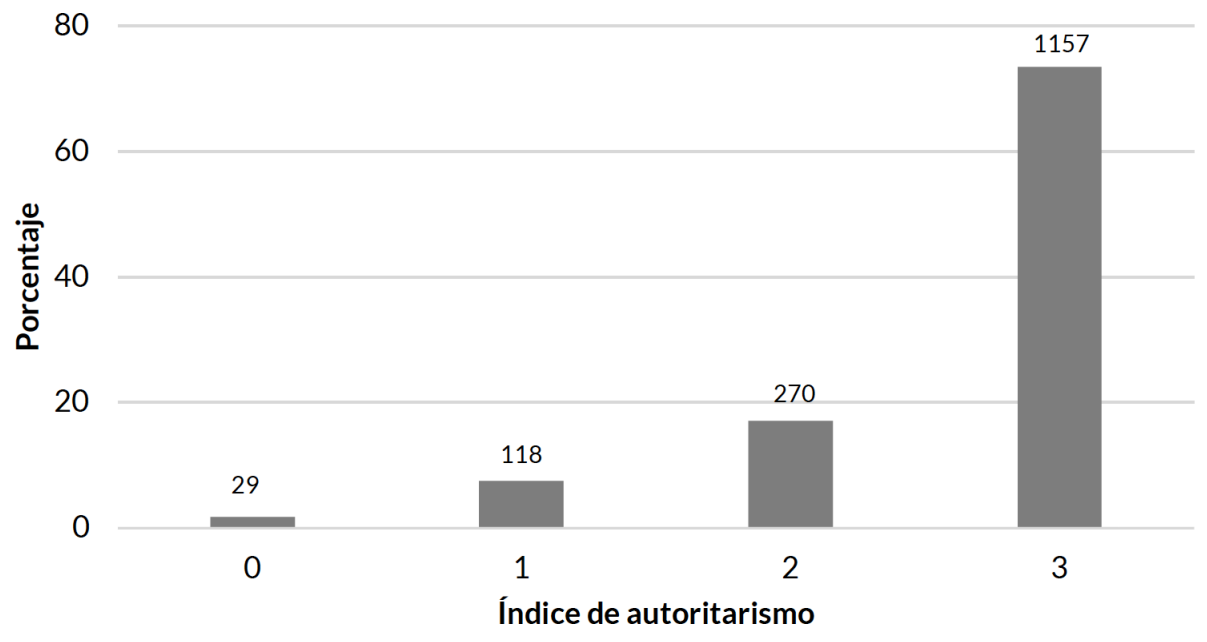

Fuente: Elaboración propia.

Como muestra la Figura 2, la gran mayoría de entrevistados (73\%) puntúa en el valor más alto de la escala. Una explicación para este porcentaje elevado es que los datos fueron capturados antes de la segunda vuelta electoral en Perú, periodo en el que la discusión política y el debate acerca de seguridad y economía eran más intensos. Otra posible explicación es que, a diferencia de similares versiones de este instrumento donde se podía recoger la opción «ambas» a cada par de opciones, en esta encuesta se les pidió a los entrevistados que escogieran una opción. Los individuos, en este instrumento, pudieron decantarse por la opción autoritaria, siendo que en otras mediciones hubieran escogido la opción intermedia.

El objetivo principal de este trabajo es poner a prueba la perspectiva de la activación mediante amenazas del efecto del autoritarismo en las simpatías hacia candidatos presidenciales en el contexto peruano. Para la activación de los efectos del autoritarismo, se introdujo un experimento en la encuesta electoral, que dividía aleatoriamente a la muestra en tres grupos ${ }^{5}$. Los entrevistados en dos de estos

5. El objetivo de la aleatorización es generar grupos estadísticamente comparables, que difieran solo en el tratamiento administrado. Para comprobar la aleatorización se realizó un chequeo de balance 
grupos leyeron una viñeta, simulando una nota informativa, una referida al tema de la delincuencia y la otra referida al tema del estancamiento económico. El tercer grupo no leyó ninguna noticia y se usa como grupo control' .

La nota informativa, que imitaba el texto de las noticias de los periódicos de circulación nacional, incluía información verídica, aunque diseñada con el propósito de presentar el lado negativo de estos temas. En el tema de la delincuencia, por ejemplo, se enfatizaban hechos violentos (como asesinatos), la pobre resolución de los delitos por parte de la policía y se concluía que los ciudadanos se sentían cada vez más inseguros. En el tema económico se destacaba el estancamiento y su efecto negativo en el consumo y en el empleo. En ambos casos, el objetivo era generar una sensación de amenaza en los entrevistados.

\section{RESULTADOS DEL EXPERIMENTO}

En primer lugar, se puede analizar la relación directa del autoritarismo con el voto hacia candidatos autoritarios, como Fujimori, y hacia candidatos no autoritarios, como Kuczynski y Mendoza. La Figura 3 muestra la proporción del voto reportado hacia cada candidato en la primera vuelta por niveles de autoritarismo.

Se muestra que, tal como se esperaba, la proporción del voto hacia la candidata autoritaria aumenta a medida que aumenta el autoritarismo de los individuos. Esta relación directa no es clara para los candidatos no autoritarios, sean de derecha, como Kuczynski, o de izquierda, como Mendoza. Se observa, además, diferencias significativas en el voto reportado hacia estos candidatos entre los más autoritarios, a favor de Keiko Fujimori.

En este trabajo, la variable dependiente no es el voto por cada uno de los candidatos, debido a que el diseño experimental se ubicó luego de estas preguntas en el cuestionario, por lo que no se pudo usar el voto reportado en la primera vuelta ni la intención de voto para la segunda vuelta como variable dependiente ${ }^{7}$. La variable dependiente que se usa en este trabajo es la simpatía hacia los candidatos, medida como el grado en el que le gusta cada candidato en una escala de 1 al 10, donde 1 significa que «no le gusta nada» y 10 que «le gusta mucho». Esta variable recoge el

\footnotetext{
entre los grupos aleatorios y se encontró que no había diferencias estadísticamente significativas con relación a edad, educación, ideología, proporción de mestizos, vivir en la región sur, nivel socioeconómico y voto a Keiko Fujimori en primera vuelta. Se encontró un desbalance en la variable proporción de mujeres. Los resultados que se muestran a continuación toman en cuenta este desbalance.

6. En el Anexo 1 se presentan los textos de cada una de las notas informativas.

7. Al tratarse de una encuesta electoral, el diseño del cuestionario requería que el reporte del voto en primera vuelta y la intención de voto en la segunda vuelta no estuvieran influenciados por un diseño experimental.
} 
Figura 3. Voto a candidatos por niveles de autoritarismo

$50 \%$

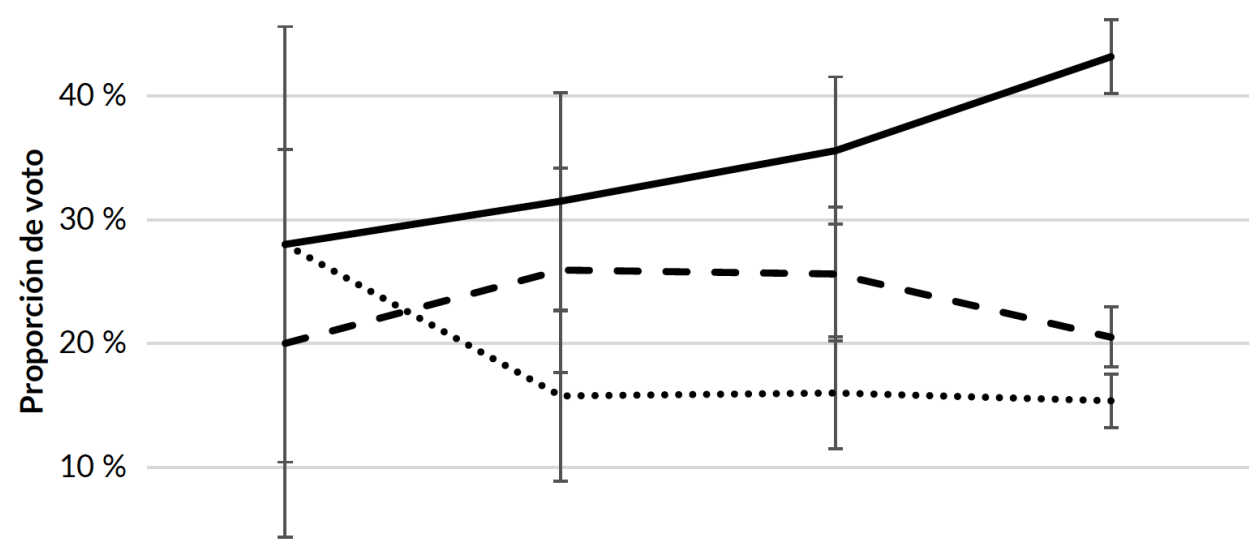

$0 \%$

0

- Voto a Fujimori _ - Voto a Kuczynski $\quad$-..... Voto a Mendoza

1

Índice de autoritarismo
2

3

Fuente: Elaboración propia.

apoyo hacia estos candidatos y está, además, muy correlacionada con el voto, y, por tanto, puede utilizarse como un buen proxy para la opción electoral8.

Se estimaron modelos de regresión de mínimos cuadrados para cada uno de los candidatos. Estos modelos incluyeron variables binarias (dummy) por cada tratamiento (la condición de control es la base de referencia) y el índice de autoritarismo como variables independientes. Adicionalmente se crearon variables interactivas multiplicando cada variable binaria de tratamiento por el índice de autoritarismo. De esta forma, los resultados de estos modelos nos indican, para cada candidato, cómo es la relación entre el autoritarismo y la simpatía por el candidato para cada grupo de tratamiento. En cada modelo se controló además por la variable mujer, por haberse detectado un desbalance en esta variable en la aleatorización?.

8. Los que manifestaron una intención de voto hacia Keiko Fujimori en la segunda vuelta puntuaban un gusto promedio de 8.52 puntos y los que reportaron una intención de voto hacia Kuczynski puntuaban un gusto promedio de 7.38. Estas mismas relaciones se verifican cuando se cruza la dirección del voto en la primera vuelta y el gusto por otros candidatos. En todos los casos, las personas califican con un puntaje más alto en gusto al candidato por el que votaron en la primera vuelta.

9. No se incorporaron otras variables de control, pues la aleatorización hace que los grupos de tratamiento y control sean estadísticamente similares, excepto en la intervención. 
La Tabla 1 muestra los resultados para la simpatía predicha a Keiko Fujimori por cada grupo de tratamiento y por niveles de autoritarismo ${ }^{10}$.

Tabla 1. Simpatía predicha hacia Keiko Fujimori por cada grupo de tratamiento y por niveles de autoritarismo

\begin{tabular}{lcccc}
\hline Grupo & Autoritarismo & Media & Error estándar & Intervalo de confianza al 95\% \\
\hline Control & 0 & 5,49 & 0,61 & {$[4,28-6,69]$} \\
& 1 & 5,55 & 0,40 & {$[4,77-6,34]$} \\
\hline Crimen & 2 & 5,62 & 0,21 & {$[5,20-6,04]$} \\
& 3 & 5,69 & 0,18 & {$[5,34-6,03]$} \\
\hline \multirow{2}{*}{ Economía } & 0 & 3,74 & 0,64 & {$[2,49-4,99]$} \\
& 1 & 4,39 & 0,41 & {$[3,58-5,20]$} \\
& 2 & 5,04 & 0,22 & {$[4,61-5,48]$} \\
& 3 & 5,69 & 0,20 & {$[5,30-6,08]$} \\
& 1 & 3,09 & 0,69 & {$[1,73-4,45]$} \\
& 2 & 3,99 & 0,45 & {$[3,10-4,88]$} \\
& 3 & 4,89 & 0,24 & {$[5,41-6,17]$} \\
\hline
\end{tabular}

Fuente: Elaboración propia.

Esta tabla muestra que la simpatía predicha a Keiko Fujimori en el grupo control no cambia significativamente a medida que aumenta el autoritarismo de los individuos. En este caso se puede decir que el autoritarismo está presente en los individuos, pero su efecto en la simpatía no ha sido activado por las amenazas. Caso contrario ocurre cuando los entrevistados son sometidos a información respecto de las amenazas económica y del crimen. Para esos individuos, a medida que aumenta el autoritarismo de los individuos, aumenta la simpatía hacia Fujimori. En el caso de la amenaza económica, se observan diferencias más marcadas en la simpatía predicha a Fujimori entre niveles de autoritarismo.

10. Los resultados completos del análisis de regresión pueden ser vistos en el Anexo 2. 
Figura 4. Valores predichos de la simpatía hacia Keiko Fujimori por índice de actitudes autoritarias y grupo de tratamiento
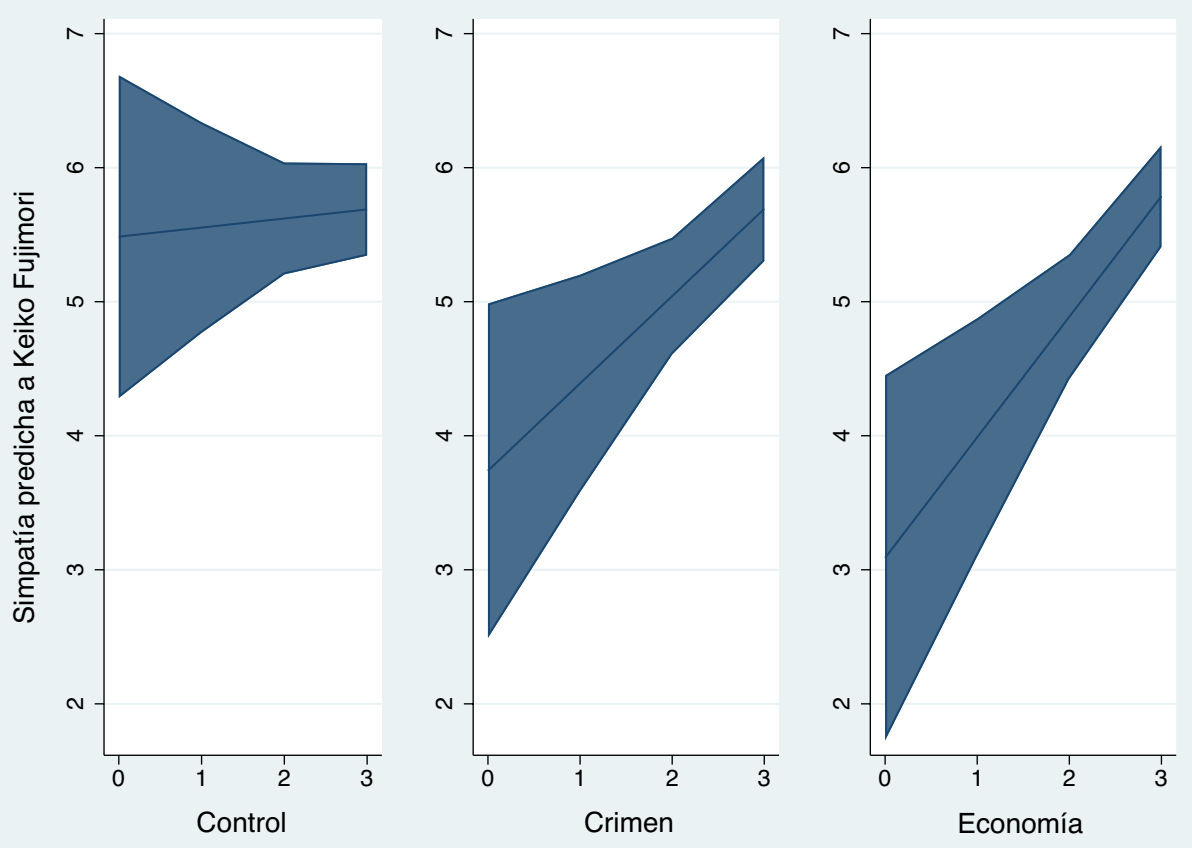

Fuente: Elaboración propia.

Los resultados de la Figura 4 muestran que los tratamientos de amenazas activan una relación positiva entre el autoritarismo y las simpatías hacia una candidata percibida por el electorado como autoritaria. Para el grupo que leyó la noticia de amenaza económica, la simpatía predicha a Fujimori aumenta 2,7 puntos en promedio, mientras que para el grupo que leyó la amenaza por el crimen, la simpatía predicha aumenta 1,95 puntos, en una escala de 1 a 10.

Estos resultados brindan evidencia a favor de la capacidad moderadora de las amenazas en la relación entre autoritarismo latente y simpatía hacia candidatos/as autoritarios/as. Este fenómeno no se halla, en cambio, cuando se trata de observar la simpatía hacia candidatos no autoritarios. Las Figuras 5 y 6 presentan los resultados para la simpatía predicha a favor de los candidatos no autoritarios - Kuczynski y Mendoza- por grupo de tratamiento y niveles de autoritarismo.

Los resultados para el candidato no autoritario muestran que los tratamientos de amenazas no generan cambios en la relación entre el autoritarismo latente y la simpatía hacia Kuczynski. Las pendientes de cambio no aumentan en los grupos de 
tratamiento, por el contrario, se observa una ligera tendencia a que disminuya la simpatía predicha, aunque estas diferencias no son significativas (las variables interactivas entre autoritarismo y las amenazas muestran un $p=0,78$ para el crimen y $\mathrm{p}=0,41$ para la economía) ${ }^{11}$.

Una situación similar se observa en el caso de Verónika Mendoza, otra candidata no autoritaria. Como se observa en la Figura 6, la simpatía predicha en favor de esta candidata cambia negativamente con los niveles de autoritarismo en todos los grupos, aunque estas diferencias no son significativas para los grupos de tratamiento ( $p=0,41$ para la amenaza económica y $p=0,36$ para la amenaza del crimen). En cambio, en el grupo control se muestra que, en ausencia de amenazas, las personas con predisposiciones autoritarias tienen una menor simpatía hacia esta candidata.

Figura 5. Valores predichos de la simpatía hacia Pedro Pablo Kuczynski por índice de actitudes autoritarias y grupo de tratamiento
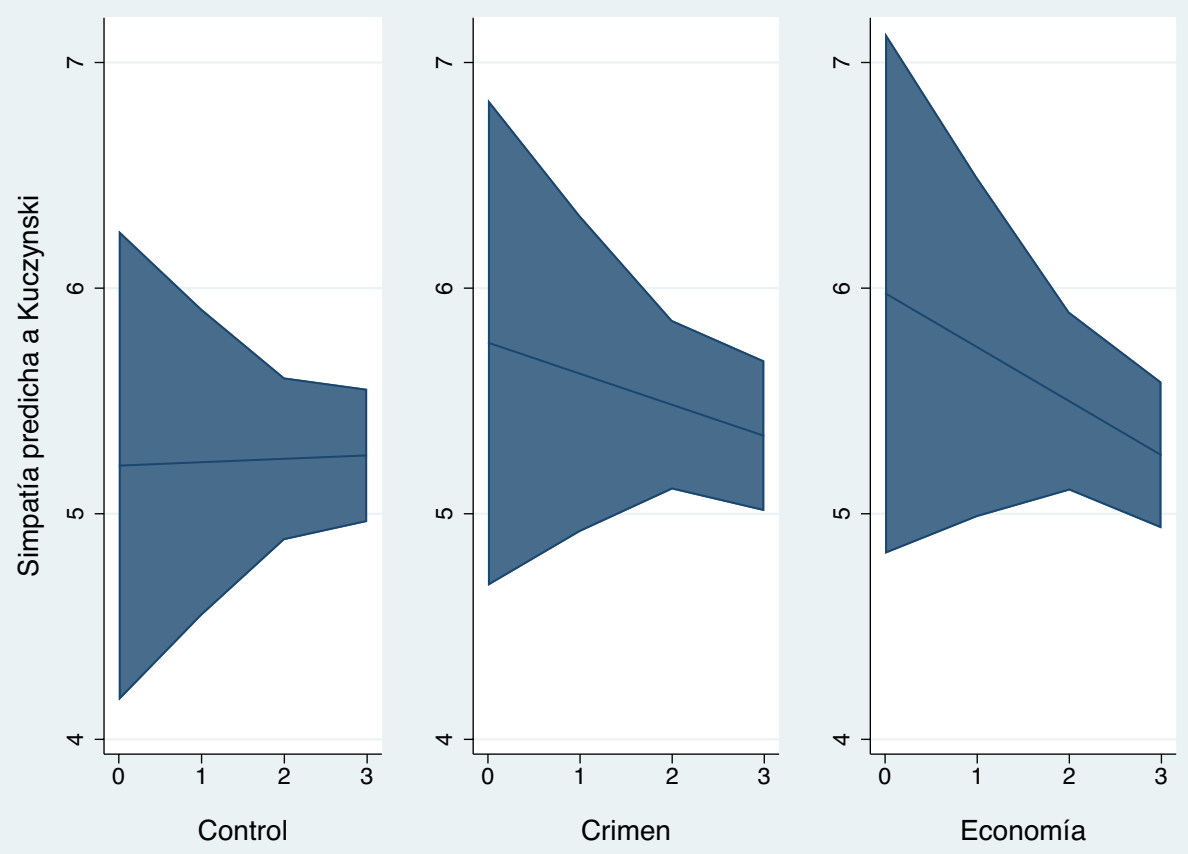

Fuente: Elaboración propia.

11. Los modelos multivariados son presentados en el Anexo 2. 
Figura 6. Valores predichos de la simpatía hacia Verónika Mendoza por índice de actitudes autoritarias y grupo de tratamiento
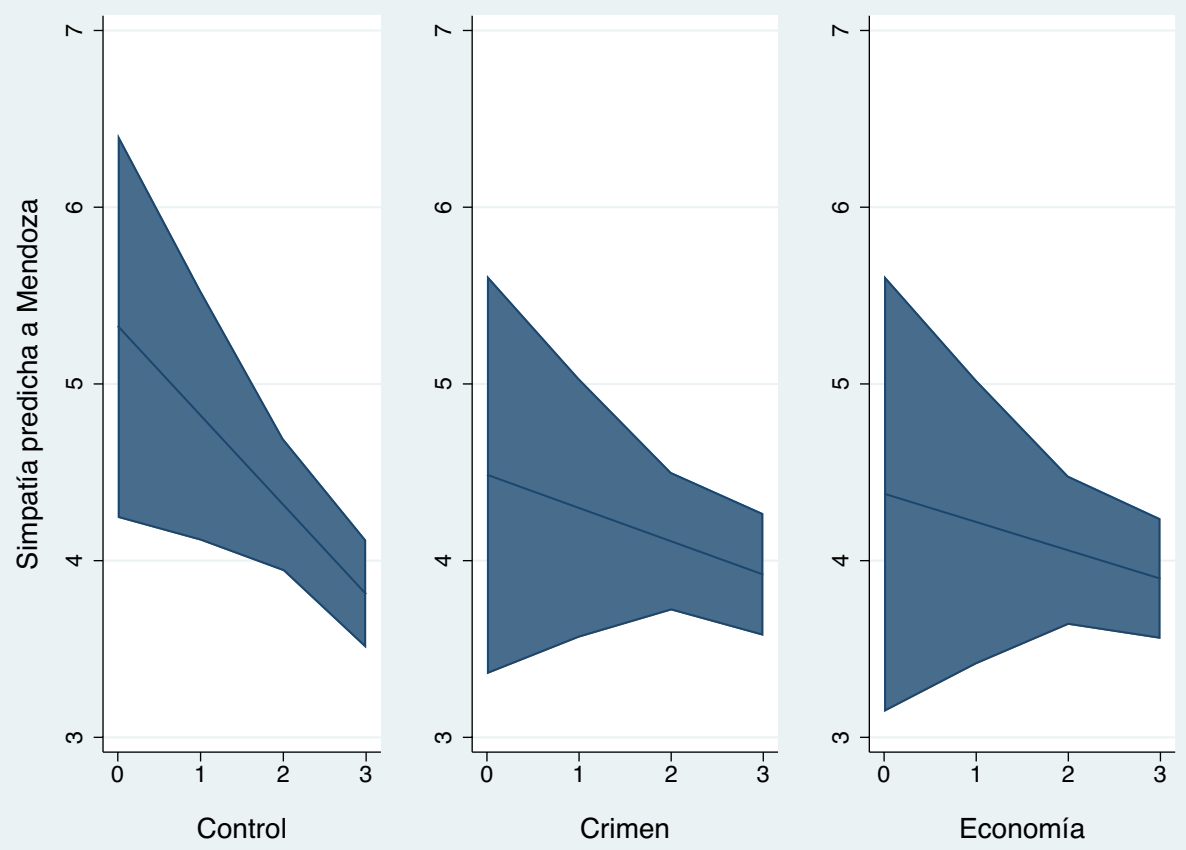

Fuente: Elaboración propia.

\section{DISCUSIÓN}

En este trabajo se encuentra que el autoritarismo individual es una variable que importa en las simpatías de los peruanos hacia candidatos presidenciales. Estos resultados a su vez son congruentes con hallazgos acerca del efecto del autoritarismo en el comportamiento electoral en América Latina: la relación entre este rasgo y el apoyo hacia candidatos de derecha autoritaria. Aquí no solo se evalúa el impacto directo del autoritarismo en el apoyo otorgado a esos candidatos, sino que se pone a prueba una perspectiva teórica que señala que las amenazas activan la relación entre el autoritarismo y la simpatía o apoyo hacia candidatos de mano dura. Este diseño experimental constituye un aporte a la literatura porque muestra que esta relación aparece o se fortalece cuando se tiene un contexto con amenazas.

Otro aporte a la discusión es la diferenciación entre tipo de amenazas. La literatura ha usado tanto amenazas económicas, políticas y amenazas a la seguridad 
como catalizadores del autoritarismo (Feldman y Stenner, 1997; Merolla y Zechmeister, 2009). Los resultados anteriores, sin embargo, son mixtos. En este trabajo proponemos dos amenazas que recurrentemente están en la agenda pública, en el Perú y en América Latina, más aún en contextos electorales: la situación económica y la situación del crimen y la delincuencia.

En este trabajo no se tenía una hipótesis preliminar acerca del efecto diferenciado de cada tipo de amenaza. Se pensaba que el crimen y la delincuencia, al haber desplazado a la economía como el principal problema de los peruanos, podría tener un efecto mayor. Sin embargo, mientras que la delincuencia es un fenómeno relativamente reciente, los problemas económicos tienen antecedentes funestos, sobre todo en la década de los ochenta y, por lo tanto, podrían haber mantenido su condición de principal amenaza al statu quo.

Empíricamente, se encuentra que las amenazas económicas activan el efecto del autoritarismo en la simpatía hacia candidatos autoritarios en mayor medida que lo hacen las amenazas respecto al crimen y delincuencia. Este hallazgo es congruente con un estudio anterior acerca de los factores de la aprobación de Alberto Fujimori en Perú. Según Weyland (2000), la popularidad de Fujimori en los noventa se debió en mayor medida al éxito en la estabilización de la economía que debido al éxito en la lucha contra el terrorismo de Sendero Luminoso. Esto indicaría que los ciudadanos peruanos tendrían una mayor sensibilidad a las amenazas económicas y premiarían en mayor medida su resolución que la atención que tienen a temas de seguridad interna. Esta diferencia, sin embargo, requiere mayor investigación.

Esto también podría sugerir un potencial efecto generacional. La cohorte que vivió las experiencias económicas deprimentes de los ochenta puede ser más susceptible a las amenazas económicas. En consecuencia, este grupo experimentaría en mayor medida la activación del efecto del autoritarismo en la simpatía hacia candidatos autoritarios.

Otro aspecto resaltante es que los peruanos exhiben niveles considerablemente altos en esta actitud, independientemente de la medida que se use. Esta elevada magnitud haría que sea más difícil encontrar relaciones significativas; sin embargo, en este trabajo se encuentra que el autoritarismo es un factor explicativo de la simpatía hacia candidatos, en particular hacia Keiko Fujimori. Es decir, si en general los peruanos muestran niveles elevados de esta característica, es posible afirmar que la diferencia, en este caso, se daría entre autoritarios (ciudadanos promedio) y muy autoritarios (simpatizantes de Fujimori).

Este diseño experimental ha permitido observar, además, que los candidatos no autoritarios, ya sean de izquierda o derecha, no reciben las simpatías de ciudadanos autoritarios, incluso bajo el efecto de amenazas. Para el caso de candidatos autoritarios, el contexto de las elecciones en Perú en el 2016 no permitió evaluar la relación del autoritarismo con un candidato de izquierda autoritaria. El candidato 
en este cuadrante -Gregorio Santos- alcanzó una votación marginal (4 \% de los votos válidos), por lo que solo el $2.3 \%$ de entrevistados reportaron votar por él en esta encuesta. Expandiendo hallazgos previos, este trabajo encuentra que los ciudadanos autoritarios bajo el efecto de amenazas registran una mayor simpatía hacia una candidata autoritaria de derecha. Sin embargo, no permite saber si este mismo efecto se observa para un candidato autoritario de izquierda. Esta hipótesis permanece empíricamente abierta.

Es necesario indicar que, según los resultados presentados, un contexto amenazador pudo haber beneficiado a Keiko Fujimori, sobre todo considerando la magnitud del autoritarismo entre los electores peruanos. Sin embargo, esta candidata perdió en la segunda vuelta. Esta aparente paradoja puede deberse a que el efecto del autoritarismo en las simpatías o en el voto puede haberse dado más claramente en la primera vuelta, contexto que se recogió en esta investigación. Para la segunda vuelta, los términos de la discusión variaron desde los aspectos de seguridad ciudadana y la economía hacia una discusión sobre el talante democrático de los candidatos Kuczynski y Fujimori. Este viraje de la discusión pudo haber aminorado el efecto del autoritarismo en favor de Fujimori y ser determinante en un contexto donde Kuczynski obtuvo la presidencia con menos del 0,25 \% del total de votos válidos de diferencia con respecto a su competidora.

Finalmente, en este trabajo se ha reportado un efecto directo y un efecto activado del autoritarismo en la simpatía hacia candidatos autoritarios. Es posible que ambos resultados sean complementarios. Una posibilidad es que el efecto directo sea una base sobre la que las amenazas operen como un moderador, amplificando el efecto. Si en el contexto peruano tenemos un grupo importante de ciudadanos en valores altos del autoritarismo, rasgo que marca sus opiniones políticas en tiempos regulares; en un contexto electoral, marcado por mensajes amenazantes, este efecto se vería exacerbado y, por lo tanto, pudiera tener un efecto mayor sobre sus opiniones, simpatías y apoyos electorales. Esta es una hipótesis que no se ha probado en este trabajo y que es una avenida para futuras investigaciones.

\section{REFERENCIAS}

Adorno, T. W., Frenkel-Brunswik, E., Levinson, D. J. y Sanford, R. N. (1950). The authoritarian personality. Harper.

Altemeyer, B. (1996). The authoritarian specter. Harvard University Press.

Azpuru, D. y Malone, M. F. T. (2018). Parenting Attitudes and Public Support for Political Authoritarianism in Latin America. International Journal of Public Opinion Research, 31(3), 570-587. https://doi.org/10.1093/ijpor/edy017.

Benhabib, S. (2013, junio 3). Turkey's Authoritarian Turn. The New York Times. www.nytimes.com/2013/06/04/opinion/turkeys-authoritarian-turn.html. 
Bugonázy, A. (2017). Illiberal democracy in Hungary: authoritarian diffusion or domestic causation. Democratization, 24(7), 1307-1325. https://doi.org/10.1080/13510347.201 7.1328676.

Carrión, J., Zárate, P., Boidi, M. F. y Zechmeister, E. J. (2018). Cultura Política de la Democracia en Perú y en las Américas, 2016/7: Un estudio comparado sobre democracia y gobernabilidad. Instituto de Estudios Peruanos.

Christiansen, A. (2016). Ideología, emociones y actitudes políticas durante las elecciones generales peruanas de 2011. [Tesis de pregrado no publicada]. Pontificia Universidad Católica del Perú.

Cohen, M. J. y Smith, A. E. (2016). Do authoritarians vote for authoritarians? Evidence from Latin America. Research and Politics, 3(4), 1-8. https://doi.org/10.1177 \%2F2053168016684066.

Costa, G. y Romero, C. (2014). ¿Quiénes son los criminales en el Perú y por qué? Factores de riesgo social e historias criminales.

Dammert, L. y Malone, M. F. T. (2008). Fear of Crime or Fear of Life? Public Insecurities in Chile. Bulletin of Latin American Research, 22(1), 79-101. https://doi. org/10.1111/1470-9856.00065.

Federico, C., Johnson, C. y Lavine, H. (2016, marzo 8). Here's why Trump's supporters tolerate his «liberal» economic positions. The Washington Post: The Monkey Cage. https:// www.washingtonpost.com/news/monkey-cage/wp/2016/03/08/heres-why-trumpssupporters-tolerate-his-liberal-economic-positions/.

Feldman, S. (2003). Enforcing social conformity: A theory of authoritarianism. Political Psychology, 24(1), 41-74. https://doi.org/10.1111/0162-895X.00316.

Feldman, S.y Stenner, K. (1997). Perceived threat and authoritarianism. Political Psychology, 18(4), 741-770. https://doi.org/10.1111/0162-895X.00077.

Fromm, E. (1941). Escape from freedom. Farrar \& Rinehart.

Gestión. (2015, agosto 27). PPK: Estas son las propuestas del equipo económico de Kuczynski. https://gestion.pe/economia/ppk-son-propuestas-equipo-economico-kuczynski-98467.

Gestión. (2016, mayo 29). Construiremos 20 cárceles para delincuentes juveniles y peligrosos. http://gestion.pe/politica/keiko-fujimori-construiremos-20-carceles-delincuentes-juveniles-y-peligrosos-2161940.

GFK. (2016). Estudio de opinión pública nacional - urbano rural. https://www.gfk.com/fileadmin/user_upload/dyna_content/PE/GfK_Opinio_n_Mayo_2016_1.pdf.

Haynes, J. (2001). Democracy in the Developing World: Africa, Asia, Latin America and the Middle East. Polity Press.

Hetherington, M. y Suhay, E. (2011). Authoritarianism, threat, and Americans' support for the war on terror. American Journal of Political Science, 55(3), 546-560. https://doi. org/10.1111/j.1540-5907.2011.00514.x.

Janowitz, M. y Marvick, D. (1953). Authoritarianism and Political Behavior. The Public Opinion Quarterly, 17(2), 185-201.

Jaramillo, F. y Zambrano, O. (2013). La clase media en Perú: cuantificación y evolución reciente. (Nota Técnica \# IDB-TN-550). Banco Interamericano de Desarrollo.

Lakoff, G. (2016, julio 22). Understanding Trump. Common Dreams. https://www.commondreams.org/views/2016/07/22/understanding-trump. 
La República. (2016, enero 5). Verónika Mendoza: «Nuestra mejor arma contra la delincuencia será la inteligencia». https://larepublica.pe/politica/731511-veronika-mendoza-nuestra-mejor-arma-contra-la-delincuencia-sera-la-inteligencia.

Lavine, H., Lodge, M. y Freitas K. (2005). Threat, Authoritarianism, and Selective Exposure to Information. Political Psychology, 26(2), 219-244. https://doi. org/10.1111/j.1467-9221.2005.00416.x.

Levitsky, S. y Way, L. A. (2010). Competitive authoritarianism: Hybrid regimes after the Cold War. Cambridge University Press.

Lolas, J. (2016, junio 3). Keiko: mano dura contra delincuentes desde el 28. La Primera. http:// www.laprimera.pe/keiko-mano-dura-contra-delincuentes-desde-el-28.

MacWilliams, M. C. (2016). Who Decides When The Party Doesn't? Authoritarian Voters and the Rise of Donald Trump. PS: Political Science \& Politics, 49(4), 716-721. https://doi. org/10.1017/S1049096516001463.

Merolla, J. L. y Zechmeister, E. J. (2009). Democracy at risk: How terrorist threats affect the public. University of Chicago Press.

Nordland, R. (2017, febrero 1). Authoritarian Leaders Greet Trump as One of Their Own. New York Times. https://www.nytimes.com/2017/02/01/world/asia/donald-trumpvladimir-putin-rodrigo-dutert-kim-jong-un.html.

Norris, P. e Inglehart, R. (2017, agosto 31-septiembre 3). Cultural backlash: Values and voting for populist authoritarian parties in Europe [Presentación en conferencia]. APSA Annual Meeting \& Exhibition, San Francisco, CA, United States.

Palacios, R. M. (2016, abril 6). ¿Por qué la oferta económica de Verónika Mendoza es peligrosa para todos? http://rosamariapalacios.pe/2016/04/06/por-que-la-oferta-economicade-veronika-mendoza-es-peligrosa-para-todos/.

Paredes, C. (2016, abril 15). La economía y las elecciones del 2016 en el Perú. Foro Económico. http://focoeconomico.org/2016/04/15/la-economia-y-las-elecciones-del-2016-en-elperu/.

Roccato, M., Vieno A. y Russo, S. (2014). The country's crime rate moderates the relation between authoritarian predispositions and the manifestations of authoritarianism: A multilevel, multinational study. European Journal of Personality, 28(1), 14-24. https://doi. org/10.1002/per.1922.

Rottenbacher de Rojas, J. M. (2012). Vigencia del continuo ideológico izquierda/derecha durante las elecciones presidenciales de 2011 en Lima-Perú. Revista de Psicología (PUCP), 30(2), 281-315.

Singer, A. J., Chouhy C., Lehmann P. S., Stevens, J. N. y Gertz, M. (2019). Economic anxieties, fear of crime, and punitive attitudes in Latin America. Punishment \& Society, 22(2), 181206. https://doi.org/10.1177\%2F1462474519873659.

Stampini, M., Robles, M., Sáenz, M., Irrabarán, P. y Medellín, N. (2015). Pobreza, vulnerabilidad y la clase media en América Latina. (IDB Working Series Papers, No. IDB-WP-591). Inter-American Development Bank (IDB).

Stenner, K. (2005). The authoritarian dynamic. Cambridge University Press.

Stevens, D., Bishin, B. G. y Barr, R. R. (2006). Authoritarian attitudes, democracy, and policy preferences among Latin American elites. American Journal of Political Science, 50(3), 606-620. https://doi.org/10.1111/j.1540-5907.2006.00204.x. 
Sulmont, D. (2018). ¿Existe el voto programático en elecciones con un sistema de partidos políticos débil? Un análisis de las elecciones presidenciales peruanas de 2016. Revista de Ciencia Política, 38(3), 429-457. http://dx.doi.org/10.4067/ S0718-090X2018000300429.

Taub, A. (2016, marzo 1). The rise of American authoritarianism. Vox. https://www.vox. com/2016/3/1/11127424/trump-authoritarianism.

Weyland, K. (2000). A Paradox of Success? Determinants of Political Support for President Fujimori. International Studies Quarterly, 44(3), 481-501. https://doi. org/10.1111/0020-8833.00168.

\section{ANEXO 1}

Nota informativa para el tratamiento de amenaza por el crimen: Miedo al aumento de la delincuencia en Perú

Todos los días vemos en los noticieros hechos de violencia e inseguridad ciudadana. A mediados de marzo pasado, delincuentes asesinaron a dos policías tras robar 10.000 soles a un cambista en el Rímac. En el Callao, a pesar del estado de emergencia decretado por el gobierno, siguen ocurriendo hechos de violencia. Sólo en el 2016 ya se han registrado 15 asesinatos en el puerto chalaco. En Trujillo, si bien la policía ha desactivado bandas como "Los Charlys de La Esperanza» y «Los Norteños», los delitos no han disminuido y Trujillo sigue siendo la segunda ciudad con mayores índices de delincuencia en el país. Según la propia Policía Nacional, solo un $15 \%$ de todos los casos criminales logran ser resueltos efectivamente.

La delincuencia y la inseguridad ciudadana son una de las principales preocupaciones de los peruanos. Si nos comparamos con otros países de América Latina, el Perú registra una de las más altas tasas de víctimas de delincuencia en la región. Varios especialistas sostienen que el Estado no ha adoptado las medidas necesarias para enfrentar este problema, por lo que la delincuencia e inseguridad podrían agravarse en los próximos años, haciendo sentir a los ciudadanos cada vez más inseguros.

\section{Nota informativa para el tratamiento de amenaza económica: Preocupación por la situación económica del Perú}

En los últimos años, los precios internacionales de las principales materias primas que exporta el Perú se han reducido en forma importante, lo que viene afectando a la economía nacional. Según varios expertos la economía peruana está entrando en un periodo de "vacas flacas»: la inversión pública y privada han 
disminuido significativamente, así como el consumo de los hogares y las empresas. Todo indica que en los próximos años el crecimiento económico será mucho menor que el esperado.

Este menor crecimiento afectará los ingresos de las familias peruanas ya que el ritmo de creación de nuevos empleos será menor, conseguir trabajo será más complicado e incluso podría aumentar el desempleo. Además, las encuestas de opinión muestran que la mayoría de los peruanos siente que la economía nacional no se está recuperando y que la situación económica de sus familias no mejorará este año. El próximo gobierno deberá enfrentar una economía con mayores problemas que en el pasado, lo que afectará el bolsillo de los peruanos.

\section{ANEXO 2}

Tabla A1. Resultados del análisis de regresión por candidato

\begin{tabular}{llll}
\hline \multicolumn{1}{c}{ Variables } & \multicolumn{1}{c}{ Gusto a Fujimori } & \multicolumn{1}{c}{ Gusto a Kuczynski } & \multicolumn{1}{c}{ Gusto a Mendoza } \\
\hline \multirow{2}{*}{ Amenaza crimen } & $-1,06$ & 0,32 & $-0,59$ \\
& $(0,83)$ & $(0,72)$ & $(0,75)$ \\
\hline \multirow{2}{*}{ Amenaza económica } & $-2,05^{*}$ & 0,66 & $-0,75$ \\
& $(0,89)$ & $(0,76)$ & $(0,80)$ \\
\hline Autoritarismo & 0,07 & 0,02 & $-0,51^{*}$ \\
\hline Crimen $x$ & $(0,22)$ & $(0,19)$ & $(0,20)$ \\
Autoritarismo & 0,34 & $-0,08$ & 0,23 \\
Economía x & $(0,31)$ & $(0,27)$ & $(0,28)$ \\
Autoritarismo & $0,71^{*}$ & $-0,23$ & 0,27 \\
\hline \multirow{2}{*}{ Mujer } & $(0,32)$ & $(0,28)$ & $(0,29)$ \\
\hline Constante & $0,76^{*}$ & $-0,29$ & $-0,48^{*}$ \\
\hline $\mathrm{N}$ & $(0,18)$ & $(0,15)$ & $(0,16)$ \\
\hline
\end{tabular}

Fuente: Elaboración propia. 